\title{
THE GROUP OF LARGE DIFFEOMORPHISMS IN GENERAL RELATIVITY
}

\author{
DOMENICO GIULINI \\ Fakultät für Physik der Universität Freiburg, \\ Hermann Herder Straße 3, D-79104 Freiburg, Germany \\ E-mail: giulini@sun2.ruf.uni-freiburg.de
}

\begin{abstract}
We investigate the mapping class groups of diffeomorphisms fixing a frame at a point for general classes of 3-manifolds. These groups form the equivalent to the groups of large gauge transformations in Yang-Mills theories. They are also isomorphic to the fundamental groups of the spaces of 3-metrics modulo diffeomorphisms, which are the analogues in General Relativity to gauge-orbit spaces in gauge theories.
\end{abstract}

1. Introduction. The dynamics of General Relativity can be cast into the form of a constraint Hamiltonian system. For this, the space-time $M$ is assumed to be a topological product $\Sigma \times \mathbf{R}$. Restricting to pure gravity, the unconstrained phase space is given by the cotangent bundle, $T^{*}(\mathcal{R})$, over the space $\mathcal{R}=\operatorname{Riem}(\Sigma)$ of Riemannian metrics on a 3-manifold $\Sigma$. Initial data thus consist of a 3-metric, $g_{a b}$, and a contravariant tensor density of weight one, $\pi^{a b}$. Once the space-time $M$ has evolved from $\Sigma, \pi^{a b}$ is related to the extrinsic curvature $K^{a b}$ of $\Sigma$ in $M$ via $\pi^{a b}=\sqrt{g}\left(K^{a b}-g^{a b} K_{c}^{c}\right)$, where $g=\operatorname{det}\left\{g_{a b}\right\}$. But the data cannot be prescribed arbitrarily: they have to satisfy the two constraints

$$
\begin{aligned}
& D^{a}:=-2 \nabla_{b} \pi^{a b}=0, \\
& H:=G_{a b c d} \pi^{a b} \pi^{c d}-\sqrt{g} R,
\end{aligned}
$$

where $\nabla$ denotes the Levi-Civita connection for $g_{a b}$ and $R$ its Ricci-scalar. $G_{a b c d}$ is a symmetric, non-degenerate bilinear form of signature type $(-,+,+,+,+,+)$, called the DeWitt metric. Explicitly it reads:

$$
G_{a b c d}=\frac{1}{2 \sqrt{g}}\left(g_{a c} g_{b d}+g_{a d} g_{b c}-g_{a b} g_{c d}\right) .
$$

1991 Mathematics Subject Classification: Primary 83C99; Secondary 57R50, 55S37.

This research was supported in parts by the Isaac Newton Institute, Cambridge (England), and the Center of Geometry and Physics at the Pennsylvania State University. I thank these institutions and the Banach Center in Warsaw for their hospitality.

The paper is in final form and no version of it will be published elsewhere. 
Upon integration over $\Sigma$ it defines a (weakly) non-degenerate form on $T^{*}(\mathcal{R})$. Its metric properties on the subspace of cotangent vectors satisfying (1.1) are quite complicated. In particular, it is neither positive definite nor even of definite signature [8]. To set up initial data we now have to

1. pick a 3-manifold $\Sigma$

2. find a pair $\left(g_{a b}, \pi^{a b}\right)$ satisfying $(1.1-2)$.

It is known that (1.1-2) have solutions on all topologies $\Sigma$ [22]. In principle we therefore have to consider all 3-manifolds.

This setting is similar to the Hamiltonian formulation of Yang-Mills theories: The space of gauge potentials $\mathcal{A}$ there corresponds to the space of metrics $\mathcal{R}$ here, and the group of gauge transformations (vertical bundle automorphisms) $\mathcal{G}$ there corresponds to the diffeomorphism group $\mathcal{D}$ here. The constraint (1.1), called the diffeomorphisms constraint, is analogous to the Gauss constraint in Yang-Mills theories. It should be read as a momentum mapping for the action of the diffeomorphism group on phase space which can be thought of as the lift of the action on configuration space:

$$
\mathcal{D} \times \mathcal{R} \rightarrow \mathcal{R}, \quad\left(\phi, g_{a b}\right) \mapsto \phi^{*} g_{a b}
$$

A curve $t \rightarrow \phi_{t}$ in $\mathcal{D}$, where $\phi_{0}=\mathrm{id}$, defines a vector field $\xi$ on $\Sigma$ by $\xi(p)=\left.\frac{d}{d t}\right|_{t=0} \phi_{t}(p)$. The infinitesimal version of (1.4) is then given by

$$
\left.\frac{d}{d t}\right|_{t=0} \phi_{t}^{*} g_{a b}=\nabla_{a} \xi_{b}+\nabla_{b} \xi_{a}
$$

where the right-hand side is just the Lie derivative expressed in terms of $\nabla$. The momentum map, $P$, applied to $\xi$, is simply obtained by contracting the canonical 1 -form (" $p d q$ ") with the right-hand side of (1.5):

$$
P(\xi)=2 \int_{\Sigma} d^{3} x \pi^{a b} \nabla_{b} \xi_{a}=\int_{\Sigma} d^{3} x D^{a} \xi_{a}+2 \int_{\partial \Sigma} d^{2} x \xi_{a} \pi^{a b} n_{b},
$$

where $d^{3} x$ and $d^{2} x$ denote coordinate volume elements and $n^{a}$ the outward pointing normal of $\partial \Sigma$. We thus see that the diffeomorphism constraint generates those diffeomorphisms for which the surface integral in (1.6) vanishes. For example, if $\Sigma$ has only a single connected boundary in an asymptotically flat region and if $\xi$ asymptotically tends to a translation or rotation, then the surface integral just corresponds to the total momentum or angular momentum respectively. The corresponding motions are not generated by the constraints. Only the asymptotically trivial ones are.

From now on we restrict $\Sigma$ to have one regular end, i.e., there exists a compact set $K \subset \Sigma$ whose complement is homeomorphic to the complement of a 3 -ball in $\mathbf{R}^{3}$. This idealizes the case of an isolated gravitational system. The regularity condition allows to 1-point compactify $\Sigma$ to a compact manifold $\bar{\Sigma}=\Sigma \cup \infty$, where the added point is called $\infty$. Although physically we are interested in $\Sigma$ it is easier, and for our purposes admissible, to carry out all constructions on $\bar{\Sigma}$. We thus take $\mathcal{R}$ to be the space of 3-metrics on $\bar{\Sigma}$, and $\mathcal{D}(\bar{\Sigma})$ its diffeomorphism group. Unless stated otherwise, we understand this group to consist of orientation preserving diffeomorphisms only. $\mathcal{R}(\bar{\Sigma})$ is topologically a positive open cone in a vector space and hence contractible. Since the diffeomorphism group 'looks' differently for different $\bar{\Sigma}$ it is given the argument to indicate this dependence. There are 
two more diffeomorphism groups that we will be interested in:

$$
\begin{aligned}
& \mathcal{D}_{\infty}(\bar{\Sigma})=\{\phi \in \mathcal{D}(\bar{\Sigma}) / \phi(\infty)=\infty\}, \\
& \mathcal{D}_{F}(\bar{\Sigma})=\left\{\phi \in \mathcal{D}_{\infty}(\bar{\Sigma}) /\left.\phi_{*}\right|_{\infty}=\mathrm{id}\right\} .
\end{aligned}
$$

Topologically we do not loose anything if we replace $\mathcal{D}(\Sigma)$ with $\mathcal{D}_{\infty}(\bar{\Sigma})$ and the asymptotically trivial diffeomorphisms of $\Sigma$ with $\mathcal{D}_{F}(\bar{\Sigma})$. These spaces are pairwise homotopy equivalent. The diffeomorphism constraint generates the identity component of asymptotically trivial diffeomorphisms, i.e. the identity component $\mathcal{D}_{F}^{0}(\bar{\Sigma})$ of $\mathcal{D}_{F}(\bar{\Sigma})$. (Generally we indicate identity components by an upper case 0 .) The Hamiltonian formalism requires to regard two points in the same orbit of the group generated by the diffeomorphism constraint as physically identical. But there is still an action of the quotient group,

$$
\mathcal{S}(\bar{\Sigma})=\mathcal{D}_{F}(\bar{\Sigma}) / \mathcal{D}_{F}^{0}(\bar{\Sigma}) .
$$

Strictly speaking it is not required by the Hamiltonian formalism to also identify $\mathcal{S}(\bar{\Sigma})$ equivalent points. This is completely analogous to the situation in Yang-Mills theories, where 'large' gauge transformations are not generated by the Gauss constraint. This might lead to interesting physical consequences in the quantum theory. An example is discussed in [5]. In the classical theory it would be appropriate to quotient by the $\mathcal{S}(\bar{\Sigma})$ action if we agreed that the functions on phase space which we want to use as observables cannot separate any two points in one orbit of $\mathcal{S}(\bar{\Sigma})$. This is in fact usually assumed, so that the classical configuration space is then given by $\mathcal{Q}(\bar{\Sigma})=\mathcal{R}(\bar{\Sigma}) / \mathcal{D}_{F}(\bar{\Sigma})$, which is in fact the base of a $\mathcal{D}_{F}(\bar{\Sigma})$-principal-fibre-bundle [3]:

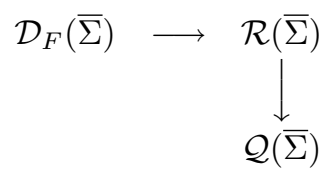

The contractibility of $\mathcal{R}(\bar{\Sigma})$ in the associated exact sequence for homotopy groups directly leads to

$$
\begin{aligned}
& \pi_{1}(\mathcal{Q}(\bar{\Sigma})) \cong \mathcal{D}_{F}(\bar{\Sigma}) / \mathcal{D}_{F}^{0}(\bar{\Sigma})=\mathcal{S}(\bar{\Sigma}) \\
& \pi_{n}(\mathcal{Q}(\bar{\Sigma})) \cong \pi_{n-1}\left(\mathcal{D}_{F}(\bar{\Sigma})\right), \quad \forall n>1
\end{aligned}
$$

Some calculations and results of higher homotopy groups (1.12) may be found in [6]. In this paper we are concerned with the groups in (1.11).

If we did not quotient by $\mathcal{S}(\bar{\Sigma})$ we would have obtained as true configuration space the universal cover $\overline{\mathcal{Q}}(\bar{\Sigma})$ of $\mathcal{Q}(\bar{\Sigma})$. A classically equivalent procedure is to work with $\overline{\mathcal{Q}}(\bar{\Sigma})$ and adding the requirement that all observables must commute with the action of $\mathcal{S}(\bar{\Sigma})$. This formulation allows us to stick with the simply connected configuration space $\overline{\mathcal{Q}}(\bar{\Sigma})$. In finite dimensions we would transfer this to the quantum theory as follows [7]: take as Hilbert space $\mathcal{H}$ the $L^{2}$-space on $\overline{\mathcal{Q}}(\bar{\Sigma})$ with some $\mathcal{S}(\bar{\Sigma})$-invariant measure. Take as algebra of observables the commutant of $\mathcal{S}(\bar{\Sigma})$. This induces a superselection structure with sectors classified by the inequivalent irreducible unitary representations of $\mathcal{S}(\bar{\Sigma})$. For this reasons, and for the obvious reasons in the classical theory, we are interested in the group $\mathcal{S}(\bar{\Sigma})$ and its representations. 
Obviously, the quantum mechanical motivation as outlined above has at least two serious problems. The first is that the infinite dimensional space $\overline{\mathcal{Q}}(\bar{\Sigma})$ is almost certainly not an appropriate measure space and therefore cannot be used to construct $\mathcal{H}$. It would have to be replaced by something else which densely contains $\overline{\mathcal{Q}}(\bar{\Sigma})$ as proper subset. This goal is pursued in the works of Ashtekar and collaborators [1]. But what persists to happen is that $\mathcal{S}(\bar{\Sigma})$ acts on the Hilbert space and is required to commute with all observables. Our motivation is therefore still valid. The second difficulty is that the discrete group $\mathcal{S}(\bar{\Sigma})$ is generically infinite and non-Abelian so that we cannot count on a general representation theory. Note that this is a major difference to the situations usually encountered in Yang-Mills theories. There the group of large gauge transformations is typically Abelian and the representation theory is simple. For example, the famous $\theta$ angle just labels the irreducible unitary representations of $\mathbf{Z}$. Some equivalents of the $\theta$-angle in quantum-gravity have been studied in the literature [11][10]. In the sequel of this paper we want to report on some approaches and results to understand the general structure of the group $\mathcal{S}(\bar{\Sigma})$.

2. 3-manifolds. We consider a general closed, connected, and oriented 3-manifold $\bar{\Sigma}$. Up to permutation of factors it can be uniquely written as a finite connected sum of prime 3-manifolds, simply called primes (see [13] for more information on the general material that follows; we denote the connected sum by $\uplus)$ :

$$
\bar{\Sigma}=\biguplus_{i=1}^{n+l} \Pi_{i}=\left(\biguplus_{i=1}^{n} P_{i}\right) \uplus\left(\biguplus_{i=1}^{l} S^{2} \times S^{1}\right),
$$

where amongst the general primes, $\Pi_{i}$, we have notationally separated the irreducible ones, $P_{i}$, from the 'handles', $S^{2} \times S^{1}$. Recall that a 3 -manifold is called irreducible if every embedded 2 -sphere bounds a 3 -ball. Irreducibility implies a trivial second homotopy group. The handle is the only reducible prime and connected sums are of course always reducible. The known primes with finite fundamental group are all of the form $S^{3} / G$ where $G$ is a finite subgroup of $S O(4)$ with proper action on $S^{3}$. The irreducible ones with infinite fundamental group are $K(\pi, 1)$ spaces, i.e., their higher-than-first homotopy groups are all trivial. Amongst those is the much better studied subclass of sufficiently large $K(\pi, 1)$ 's, i.e. those which contain incompressible surfaces. Simple examples are those of the form $S^{1} \times R_{g}$, where $R_{g}$ is a genus $g$ surface, or the six orientable flat 3manifolds. It is conjectured but unproven that all primes with finite fundamental group are covered by $S^{3}$ and all the irreducible ones with infinite fundamental group by $R^{3}$. If the Poincaré conjecture were true the former (but not the latter) would be implied. For convenience we shall in the sequel eliminate the uncertainty caused by the open Poincaré conjecture and restrict to primes not covered by homotopy spheres and their connected sums only. In (2.1) each $P_{i}$ is connected to the rest of $\bar{\Sigma}$ along a 2 -sphere, $S_{i}, 1 \leq i \leq n$, whereas each handle is connected along two 2 -spheres, $S_{i j}, 1 \leq i \leq l, j=1,2$, which should be thought of as the boundaries of the cylinder $[0,1] \times S^{2}[16][9]$.

The fundamental group of the connected sum is given by the free product of the individual ones: 


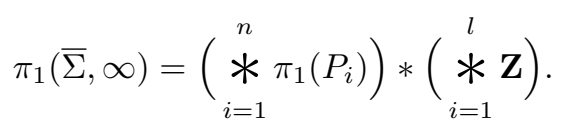

It is useful to visualize $\bar{\Sigma}$ in terms of the connected sum (2.1). Roughly speaking, $\bar{\Sigma}$ is divided into the regions interior to the primes (called interior regions) and their complement (called exterior region). The interior regions are connected to the exterior region along the pairwise disjoint 2-spheres $S_{i}$ and $S_{i j}$. This is explained in more detail in [16][9]. This allows to divide the diffeomorphisms into three different types:

1. those with support in the interior region (called interior diffeomorphisms),

2. those which leave the exterior and interior region setwise invariant (called exterior diffeomorphisms), like the exchange of two diffeomorphic primes,

3. special diffeomorphisms not in 1. and 2., called exterior slides.

These will be explained below.

An important tool in analysing $\mathcal{S}(\bar{\Sigma})$ is the following homomorphism

$$
h_{\infty}(\bar{\Sigma}): \mathcal{D}_{\infty}(\bar{\Sigma}) / \mathcal{D}_{\infty}^{0}(\bar{\Sigma}) \rightarrow \operatorname{Aut}\left(\pi_{1}(\bar{\Sigma}, \infty)\right),
$$

which one obtains by composing the loop $\gamma$ that represents the element in the homotopy group with $\phi \in \mathcal{D}_{\infty}(\bar{\Sigma})$ to $\phi \circ \gamma$. (This also works for higher homotopy groups.) Useful information on $\mathcal{S}(\bar{\Sigma})$ is then obtained by investigating the kernel and image of $h_{\infty}(\bar{\Sigma})$. For later notational convenience we also introduce the map $h_{F}$ which is defined just like $h_{\infty}$ but with $\mathcal{D}_{F} / \mathcal{D}_{F}^{0}$ replacing $\mathcal{D}_{\infty} / \mathcal{D}_{\infty}^{0}$.

Let us describe some of the important diffeomorphisms. First we turn to the internal diffeomorphisms. Take an inner collar neighbourhood of $S_{i}$, and define a diffeomorphisms by a relative $2 \pi$-rotation of its boundary 2 -spheres, and extend it to the rest of the manifold by the identity. This is called a rotation of $P_{i}$. If this diffeomorphism is not isotopic to the identity within the class of inner diffeomorphisms of $P_{i}$ holding $S_{i}$ fixed, we call $P_{i}$ spinorial. Amongst the known primes all but the lens spaces and the handle are spinorial and a connected sum is spinorial iff at least one prime is [9]. For spinorial manifolds $\mathcal{S}(\bar{\Sigma})$ is a central $\mathbf{Z}_{2}$-extension of $\mathcal{D}_{\infty}(\bar{\Sigma}) / \mathcal{D}_{\infty}^{0}(\bar{\Sigma})$. In the non-spinorial case they are isomorphic [6]. The same relation hold for the kernels of $h_{F}(\bar{\Sigma})$ and $h_{\infty}(\bar{\Sigma})$. Similarly, for a handle we can define an internal diffeomorphism by a relative $2 \pi$-rotation of $S_{i 1}$ and $S_{i 2}$, extended by the identity. This is called a twist of the $i^{\prime}$ th handle. It is not isotopic to the identity and generates $\mathcal{S}\left(S^{2} \times S^{1}\right)$. Rotations of spinorial primes and twists each generate a $\mathbf{Z}_{2}$ subgroup of $\mathcal{S}(\bar{\Sigma})$. Moreover, since they rotate parallel to 2spheres, they are in the kernel of $h_{\infty}(\bar{\Sigma})$. Clearly any two internal diffeomorphisms of different primes commute. Hence there is a homomorphism of the direct product of all $\mathcal{S}\left(\Pi_{i}\right)$ into $\mathcal{S}(\bar{\Sigma})$. Hendriks and McCullough have proven that this homomorphism is in fact an injection [15]:

$$
I: \prod_{i=1}^{n} \mathcal{S}\left(P_{i}\right) \times \mathbf{Z}_{2}^{l} \hookrightarrow \mathcal{S}(\bar{\Sigma}) .
$$

Applying a more general result of McCullough's [17] to the present situation shows that 
the kernel of $h_{F}(\bar{\Sigma})$ lies in the image of the map $I$, that is

$$
\operatorname{ker}\left(\left(h_{F}(\bar{\Sigma})\right)=\left(\prod_{i=1}^{l} \mathbf{Z}_{2}\right) \times \prod_{i=1}^{n} K_{i},\right.
$$

where $K_{i}$ denotes the kernel of $h_{F}$ in $\mathcal{S}\left(P_{i}\right)$, which, we recall, is a $\mathbf{Z}_{2}$-extensions of the kernel of $h_{\infty}\left(P_{i}\right)$ in $\mathcal{D}_{\infty}\left(P_{i}\right) / \mathcal{D}_{\infty}^{0}\left(P_{i}\right)$ if $P_{i}$ is spinorial, and isomorphic otherwise. On the other hand, suppose two diffeomorphisms $d, d^{\prime} \in \mathcal{D}_{\infty}\left(P_{i}\right)$ have the same image under $h_{\infty}\left(P_{i}\right)$. Irreducible primes have $\pi_{2}=0$ and those of the form $S^{3} / G$ have $\pi_{3}=\mathbf{Z}$ whereas $K(\pi, 1)$ primes have $\pi_{3}=0$. This implies that $d$ and $d^{\prime}$ are homotopic (see e.g. Lemma 5.1 in [21]). But for $P_{i}$ for which homotopy implies isotopy - we call it the HI-property and manifolds with this property HI-manifolds - this implies the triviality of $K_{i}$ for nonspinorial $P_{i}$ and $K_{i} \cong Z_{2}$ if $P_{i}$ is spinorial. No non-HI-prime is known so far (though non-HI connected sums! [4]). But some cases are undecided and we refer to Theorem A1 in [6] for a list of which primes have so far been proven to be of HI type. For them we have the

Proposition 1. Let $\bar{\Sigma}$ be composed of HI-primes of which $k$ are spinorial. Then $\operatorname{ker}\left(h_{F}(\bar{\Sigma})\right)=\prod_{i=1}^{k+l} \mathbf{Z}_{2}$, generated by rotations of spinorial primes and twists of handles.

The external diffeomorphism which we have to mention are the exchanges of diffeomorphic primes and the spins of handles. The latter just correspond to a mutual exchange of the two handle-ends. More details are given in [9].

Next we describe slide diffeomorphisms: Given a loop $\gamma:[0,1] \ni s \rightarrow \bar{\Sigma}$ with toroidal tubular neighbourhood $T \subset \bar{\Sigma}$ coordinatized by $(r, \theta, \varphi) \in[0,2] \times[0,2 \pi) \times[0,2 \pi)$, where $r$ is a radial coordinate parametrizing coaxial 2-tori $T_{t}=\{r=t\}$ with axis $T_{0}=\gamma$. $\theta$ and $\varphi$ coordinatize the toroidal latitudes and longitudes respectively. Let $\lambda: \mathbf{R} \rightarrow[0,1]$ be a $C^{\infty}$ step function: $\lambda(x)=0$ for $x \leq 0, \lambda(x)=1$ for $x \geq 1$. We define a 1 -parameter family of diffeomorphisms $\phi_{t}$ of $\bar{\Sigma}$ as follows:

$$
\phi_{t}(p)= \begin{cases}p & \text { for } p \in \bar{\Sigma}-T, \\ (r, \theta, \varphi+t 2 \pi \lambda(2-r)) & \text { for } p=(r, \theta, \varphi) \in T .\end{cases}
$$

The diffeomorphism $\phi_{1}=\phi_{t=1}$ is the identity inside $T_{1}$. It is called a slide along $\gamma$. Suppose $\infty$ is on $\gamma$. Then $\phi_{1} \in \mathcal{D}_{F}(\bar{\Sigma})$ and the action of $\phi_{1}$ on $\pi_{1}(\bar{\Sigma}, \infty)$ is by conjugation with $[\gamma]$, the class of $\gamma$ in $\pi_{1}(\bar{\Sigma}, \infty)$. This shows that

$$
\operatorname{Inn}\left(\pi_{1}(\bar{\Sigma}, \infty)\right) \subset \operatorname{Im}\left(h_{\infty}(\bar{\Sigma})\right),
$$

where we could have replaced $h_{\infty}$ with $h_{F}$. Next suppose we slightly change the construction above in that one of the spheres $S_{i}\left(\right.$ or $\left.S_{i j}\right)$ now sits inside $T_{1}$. We then call $\phi_{1}$ a slide of $P_{i}$ (or the $j$ 'th end of handle $i$ ) along $\gamma$. This diffeomorphism is isotopic to the identity if $\gamma$ is contractible, but not otherwise [16].

An important theorem now states that $\mathcal{S}(\bar{\Sigma})$ is generated by internal, exchange, and slide diffeomorphisms. The slides we need to consider are slides of primes or handle-ends along non-contractible loops. Consequently, $\mathcal{S}(\bar{\Sigma})$ is finitely generated if each $\mathcal{S}\left(P_{i}\right)$ is. Lucid proofs of these important results may be found in [16].

The individual groups $\mathcal{S}\left(P_{i}\right)$ have been calculated for many irreducible primes. See [21] for the spherical primes $S^{3} / G$. In the next section we show how to obtain a general 
result for the class of sufficiently large $K(\pi, 1)$ primes.

3. $\mathcal{S}(P)$ for $P$ sufficiently large $K(\pi, 1)$. Let for the moment $P$ be an irreducible prime. The spaces $\mathcal{D}_{\infty}(P)$ and $\mathcal{D}(P)$ are related by the fibration

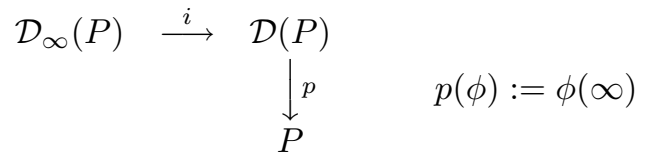

whose associated exact sequence for homotopy groups ends with (dropping the arguments $P$ for the moment)

$$
\cdots \rightarrow \pi_{1}\left(\mathcal{D}_{\infty}\right) \rightarrow \pi_{1}(\mathcal{D}) \stackrel{p_{*}}{\rightarrow} \pi_{1} \stackrel{\partial_{*}}{\rightarrow} \pi_{0}\left(\mathcal{D}_{\infty}\right) \stackrel{i_{*}}{\rightarrow} \pi_{0}(\mathcal{D}) \rightarrow 1 .
$$

The map $\partial_{*}$ is as follows: Take a class $[\gamma] \in \pi_{1}(P, \infty)$ and a representing loop, $\gamma_{t}$, at $\infty$. Take a lift, $\phi_{t}$, of $\gamma_{t}$ in $\mathcal{D}_{\infty}$ starting at the identity map, $\phi_{0}=\mathrm{id}$, so that $\phi_{t}(\infty)=\gamma_{t}$. The end point, $\phi_{1}$, defines a class $\left[\phi_{1}\right] \in \pi_{0}\left(\mathcal{D}_{\infty}\right):=\mathcal{D}_{\infty} / \mathcal{D}_{\infty}^{0}$, and one defines $\partial_{*}([\gamma]):=\left[\phi_{1}\right]$. This is indeed a well defined map since it is independent of the representative $\gamma_{t}$ of $[\gamma]$.

The lift $\phi_{t}$ may of course just be given by a slide along $\gamma$, as defined in (2.3). As noted above, $h_{\infty}\left(\left[\phi_{1}\right]\right)$ just corresponds to conjugation by $[\gamma]$, so that $h_{\infty} \circ \partial_{*}=\mathrm{Ad}$, where $\mathrm{Ad}$ denotes the 'adjoint-homomorphism' from $\pi_{1}$ to $\operatorname{Inn}\left(\pi_{1}\right)$. Its kernel is $C_{\pi}$, the centre of $\pi_{1}$. Exactness of (3.2) implies that the image of $p_{*}$ lies within that kernel:

$$
\operatorname{Im} p_{*} \subset C_{\pi} .
$$

This allows to write the inner automorphisms as a double quotient

$$
\operatorname{Inn}\left(\pi_{1}\right) \cong \frac{\pi_{1}}{\operatorname{Im} p_{*}} / \frac{C_{\pi}}{\operatorname{Im} p_{*}}=: q\left(\pi_{1} / \operatorname{Im} p_{*}\right),
$$

where $q$ just denotes the quotient map with respect to $C_{\pi} / \operatorname{Im} p_{*}$. Let $h: \mathcal{D} / \mathcal{D}^{0} \rightarrow \operatorname{Out}\left(\pi_{1}\right)$ be the non-based version of $h_{\infty}$. Diagram (3.2) can now be completed by the maps $q, h_{\infty}$ and $h$ with two commutative squares:

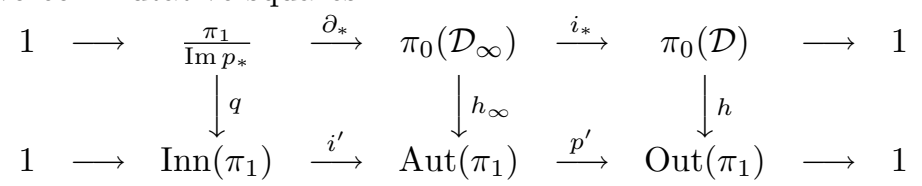

Commutativity of the left square follows from the discussion above whereas commutativity of the right square is obvious. (3.3) implies that $q$ is surjective which implies for the diagram that $h_{\infty}$ is surjective if and only if $h$ is. If we now restrict $P$ to be an irreducible HI-prime we can show

LEMMA 1. Let $P$ be an irreducible HI-prime, then $q$ is an isomorphism.

Proof. For irreducible HI primes $h_{\infty}$ is injective. From (3.5) $h_{\infty} \circ \partial_{*}=i^{\prime} \circ q$ with injective maps $h_{\infty}, \partial_{*}$ and $i^{\prime}$. So $q$ must be injective. But $q$ is a quotient map with respect to $C_{\pi} / \operatorname{Im} p_{*}$. So $\operatorname{Im} p_{*}=C_{\pi}$ and $q$ is an isomorphism.

Hatcher has proven [12] that for sufficiently large $K(\pi, 1)$ primes (which all have the $\mathrm{HI}$ property) $h$ is an isomorphism if $\mathcal{D}$ includes orientation reversing diffeomorphisms (if existent). Generalizing for the moment to this case, diagram (3.5) then implies that $h_{\infty}$ 
is also an isomorphism. Taken together with the fact that $K(\pi, 1)$ primes are spinorial (this is e.g. shown in [14] or [20]) this implies

Proposition 2. Let $P$ be a sufficiently large $K(\pi, 1)$ prime. Then $\mathcal{S}(P)$ is a central $\mathbf{Z}_{2}$ extension of $\mathrm{Aut}^{+}\left(\pi_{1}(P)\right)$.

Here we had to account for the possibility that $P$ allows for orientation reversing diffeomorphisms all of which act non-trivially on the fundamental group. Since diffeomorphisms in $\mathcal{D}_{F}(P)$ are necessarily orientation preserving, we can in this case only reach an index 2 subgroup of $\operatorname{Aut}\left(\pi_{1}(P)\right)$ which we denoted by an upper case + sign. This difference does not exist if $P$ does not allow for orientation reversing diffeomorphisms (this is the generic case, see e.g. [9] for collective information on this point), or if there exists an orientation reversing diffeomorphism acting trivially on the fundamental group, like e.g. for the handle. For example, for the 3 -torus $T^{3}$ we have $\operatorname{Aut}\left(\mathbf{Z}^{3}\right)=G L(3, \mathbf{Z})$, $\operatorname{Aut}^{+}\left(\mathbf{Z}^{3}\right)=S L(3, \mathbf{Z})$ and one may show that $\mathcal{S}\left(T^{3}\right)$ is given by the Steinberg group $S t(3, \mathbf{Z})$ (see [19] for more information on the Steinberg groups).

4. General Remarks. For a physicist it is tempting to think of the manifold $\bar{\Sigma}$ as being composed of elementary objects, the primes, just like a collection of $N=n+l$ particles from $d$ different species each with its own characteristic internal symmetry group $G_{r}, 1 \leq r \leq d$ (in doing this we think of the spin of a handle as internal diffeomorphism). In this analogy diffeomorphic primes correspond to particles of one species and the 'internal' symmetry groups $G_{r}$ to the groups $\mathcal{S}\left(\Pi_{r}\right)$. Let there be $n_{r}$ primes in the $r$ 'th diffeomorphism class so that $\sum_{r=1}^{d} n_{r}=N$. In the particle picture the total symmetry group would be a semidirect product of the internal symmetry group $G^{I}$ with the external symmetry group $G^{E}$. These are given by:

$$
\begin{aligned}
G^{I} & =\prod_{i=1}^{n_{1}} G_{1} \times \cdots \times \prod_{i=1}^{n_{d}} G_{d}, \\
G^{E} & =S_{n_{1}} \times \cdots \times S_{n_{d}} .
\end{aligned}
$$

There is a homomorphism $\theta: G^{E} \rightarrow \operatorname{Aut}\left(G^{I}\right)$, defined by $\theta=\theta_{1} \times \cdots \times \theta_{d}$, where

$$
\begin{aligned}
\theta_{j}: S_{n_{j}} & \rightarrow \operatorname{Aut}\left(\prod_{i=1}^{n_{j}} G_{j}\right) \\
\sigma & \mapsto \theta_{j}(\sigma):\left(g_{1}, \ldots, g_{n_{j}}\right) \mapsto\left(g_{\sigma(1)}, \ldots, g_{\sigma\left(n_{j}\right)}\right) .
\end{aligned}
$$

The semidirect product $G^{I} \times{ }_{\theta} G^{E}=: G^{P}$, which we call the particle group, is now defined via the multiplication law as follows: Let $\gamma_{j} \in \prod_{i=1}^{n_{j}} G_{j}, j=1, \ldots, d$, then

$$
\begin{aligned}
& \left(\gamma_{1}^{\prime}, \ldots, \gamma_{d}^{\prime} ; \sigma_{1}^{\prime}, \ldots, \sigma_{d}^{\prime}\right)\left(\gamma_{1}, \ldots, \gamma_{d} ; \sigma_{1}, \ldots, \sigma_{d}\right) \\
& \quad=\left(\gamma_{1}^{\prime}\left[\theta_{1}\left(\sigma_{1}^{\prime}\right)\left(\gamma_{1}\right)\right], \ldots, \gamma_{d}^{\prime}\left[\theta_{d}\left(\sigma_{d}^{\prime}\right)\left(\gamma_{d}\right)\right] ; \sigma_{1}^{\prime} \sigma_{1}, \ldots, \sigma_{d}^{\prime} \sigma_{d}\right) .
\end{aligned}
$$

From the discussion in Section 2 it is clear that the group $G^{P}$ is a subgroup of $\mathcal{S}(\bar{\Sigma})$ generated by internal and external diffeomorphisms. However, we also had to consider slides which were neither internal nor external and which are not compatible with the particle picture just used. As regards our understanding of $\mathcal{S}(\bar{\Sigma})$, it seems that one of 
the most interesting questions is to ask for the rôle of slides in building up $\mathcal{S}(\bar{\Sigma})$. For example, we may ask for the normal subgroup $N_{S}$ generated by slides and whether it overlaps with the particle group $G^{P}$, i.e., whether $N_{S} \cap G^{P} \neq\{1\}$. If so, the quotient $\mathcal{S}(\bar{\Sigma}) / N_{S}$ will be a non-trivial factor group of $G^{P}$. Otherwise $\mathcal{S}(\bar{\Sigma})$ will be a semidirect product of $N_{S}$ with $G^{P}$. In the sequel of this paper we attempt to answer this question.

5. Examples. In this section we wish to develop some feeling for the question just posed by presenting two examples of manifolds each consisting of a $N>2$ fold connected sum of diffeomorphic primes. In the first example the primes will be handles:

$$
\bar{\Sigma}=\biguplus_{i=1}^{N} S^{2} \times S^{1},
$$

so that the fundamental group of $\bar{\Sigma}$ is just the free group on $N$ generators $g_{1}, \ldots, g_{N}$ :

$$
\pi_{1}(\bar{\Sigma})=\underset{i=1}{*} \mathbf{Z}=F_{N} .
$$

We represent each $g_{i}$ by a loop based at $\infty$ which enters the $i$ 'th handle through $S_{i 1}$ and leave it through $S_{i 2}$ (call it the positive direction). The group $\operatorname{Aut}\left(F_{N}\right)$ can be generated by the four operations

$$
\begin{aligned}
P:\left[g_{1}, g_{2}, g_{3}, \ldots, g_{N}\right] & \mapsto\left[g_{2}, g_{1}, g_{3}, \ldots, g_{N}\right] \\
Q:\left[g_{1}, g_{2}, g_{3}, \ldots, g_{N}\right] & \mapsto\left[g_{2}, g_{3}, g_{4}, \ldots, g_{N}, g_{1}\right] \\
O:\left[g_{1}, g_{2}, g_{3}, \ldots, g_{N}\right] & \mapsto\left[g_{1}^{-1}, g_{2}, g_{3}, \ldots, g_{N}\right] \\
U:\left[g_{1}, g_{2}, g_{3}, \ldots, g_{N}\right] & \mapsto\left[g_{1} g_{2}, g_{2}, g_{3}, \ldots, g_{N}\right]
\end{aligned}
$$

whose interpretation is as follows: $P$ exchanges handle no. 1 and handle no. 2, $Q$ exchanges all $N$ handles in cyclic order, $O$ spins handle no. 1 , and $U$ slides the second end of the first handle through the second handle in a negative direction. This means that by inspection we have just proven that in the present case the map $h_{\infty}(\bar{\Sigma})$ in $(2.3)$ is surjective. From (2.5) we know that its kernel and hence that $\mathcal{S}(\bar{\Sigma})$ is an extension of $\operatorname{Aut}\left(F_{N}\right)$ by $\prod_{i=1}^{N} \mathbf{Z}_{2}$. It is also easy to describe the extension explicitly. First of all, $P$ and $Q$ alone generate the permutation group $S_{N}$ of $N$ objects which is a sub- but no factor-group of $\operatorname{Aut}\left(F_{N}\right)$, and $P, Q, O$ generate a subgroup of order $2^{N} N$ ! whose interpretation is that of a particle group with internal $\mathbf{Z}_{2}$ symmetry (the spin). A presentation of the full group is obtained by adding a generator $T$ which generates a twist on the first handle. It commutes with $U$ and $O$, squares to the identity, and has the same relations with $P, Q$ as $O$ has. Since we are given an explicit presentation of $\operatorname{Aut}\left(F_{N}\right)$ in terms of $P, Q, O, U[2]$ we can now give an explicit presentation of $\mathcal{S}(\bar{\Sigma})$ in terms of $P, Q, O, T, U$. We use the symbol $V$ to denote either $O$ or $T$ so that a relation containing $V$ is meant to be valid with $V=O$ and $V=T . A \leftrightarrow B$ says that $A$ commutes with $B$ :

$$
\begin{aligned}
& P^{2}=V^{2}=E \\
& (Q P)^{N-1}=Q^{N} \\
& P \leftrightarrow Q^{-i} P Q^{i}, \quad \text { for } 2 \leq i \leq N / 2
\end{aligned}
$$




$$
\begin{aligned}
& V \leftrightarrow Q^{-1} P Q \\
& V \leftrightarrow Q P \\
& (P V)^{4}=E \\
& T \leftrightarrow O \\
& U \leftrightarrow T \\
& U \leftrightarrow Q^{-2} P Q^{2} \quad \text { for } N>3 \\
& U \leftrightarrow Q P Q^{-1} P Q \\
& U \leftrightarrow Q^{-2} O Q^{2} \\
& U \leftrightarrow Q^{-2} U Q^{2} \quad \text { for } N>3 \\
& U \leftrightarrow O U O \\
& U \leftrightarrow P Q^{-1} O U O Q P \\
& U \leftrightarrow P Q^{-1} P Q P U P Q^{-1} P Q P \\
& \left(P Q^{-1} U Q\right)^{2}=U Q^{-1} U Q U^{-1} \\
& U^{-1} P U P O U O P O=E
\end{aligned}
$$

Most of the $\leftrightarrow$ relations have a trivial meaning. For example, (5.4j) just says that $U$ commutes with a cyclic permutation of $g_{i}$ 's for $i>2$. But this is obvious since their supports may be chosen to be disjoint. The most remarkable relation is (5.4q) which can be rewritten in the form $O P=U^{-1}\left(P U P^{-1}\right)\left(O U O^{-1}\right)$. Hence $O P$ is in the normal subgroup generated by slides. Adding the relation $U=E$ will therefore not lead to the particle group. In fact we have

THEOREM 1. The quotient with respect to the normal subgroup generated by slides is $\mathbf{Z}_{2} \times \mathbf{Z}_{2}$ generated by $T$ and $P$.

Proof. Setting $U=E$ in (5.4q) leads via (5.4a) to $P=O$. (5.4e) for $V=O$ then implies $P \leftrightarrow Q$ and (5.4b) implies $Q=P^{N-1}$, i.e., $Q=E$ for $N$ odd and $Q=P$ for $N$ even. We are thus left with two involutive commuting generators $T$ and $P$.

By simply checking the relations one may moreover prove [9]

THEOREM 2. Given a representation $\rho$ of $\mathcal{S}(\bar{\Sigma})$, the following conditions are equivalent:

1. $\rho$ is Abelian,

2. slides are represented trivially,

3. $\rho$ correlates $P$ and $O$, i.e. $\rho(P)=\rho(O)$,

4. slides and exchanges commute under $\rho$.

As regards this theorem we mention that the case $N=2$ behaves rather differently [9].

In our second example we take as prime the real projective space $R P^{3}$. The setting is very similar to the previous case so that we can be brief. The fundamental group is the free product on $N$ involutive generators $g_{1}, \ldots, g_{N}, g_{j}^{2}=E$. There is no non-trivial internal diffeomorphism (e.g. [21]), i.e. $\mathcal{S}\left(P R^{3}\right)=E$ so that the kernel of $h_{\infty}(\bar{\Sigma})$ trivial. $\mathcal{S}(\bar{\Sigma})$ is generated by the operations:

$$
P:\left[g_{1}, g_{2}, g_{3}, \ldots, g_{N}\right] \mapsto\left[g_{2}, g_{1}, g_{3}, \ldots, g_{N}\right],
$$




$$
\begin{aligned}
& Q:\left[g_{1}, g_{2}, g_{3}, \ldots, g_{N}\right] \mapsto\left[g_{2}, g_{3}, g_{4}, \ldots, g_{N}, g_{1}\right], \\
& U:\left[g_{1}, g_{2}, g_{3}, \ldots, g_{N}\right] \mapsto\left[g_{2}^{-1} g_{1} g_{2}, g_{2}, g_{3}, \ldots, g_{N}\right],
\end{aligned}
$$

and the relations are given by

$$
\begin{aligned}
& P^{2}=U^{2}=E \\
& (Q P)^{N-1}=Q^{N}=E \\
& P \leftrightarrow O^{-i} P Q^{i}, \quad \text { for }, 2 \leq i \leq N / 2 \\
& U \leftrightarrow Q^{-2} P Q^{2}, \quad \text { for }, N>3 \\
& U \leftrightarrow Q P Q^{-1} P Q \\
& U \leftrightarrow Q^{-2} U Q^{2} \\
& U \leftrightarrow Q^{-1} P U P Q \\
& Q^{-1} U Q U Q^{-1} U Q=P Q^{-1} U Q P U P Q^{-1} U Q P, \quad \text { for } N \geq 3 .
\end{aligned}
$$

Of course, the first three relations again imply that $P, Q$ generate the permutation group $S_{N}$ which here is identical to the particle group $G^{P}$. All $\leftrightarrow$ relations can be understood from simple arguments saying that the diffeomorphisms representing the left and right sides can be chosen to have disjoint support. Relation (5.6h) is less obvious. If $\mu_{i j}$ represents the unique slide of prime $j$ through prime $i$, then it can be expressed in the form: $\mu_{31} \mu_{21} \mu_{31}=\mu_{32} \mu_{21} \mu_{32}$. (Relation (5.4p) has a similar interpretation using slides of handle-ends.) In particular, it is a relation purely amongst slides. Setting $U=E$ in (5.6h) leads to no additional relations for $P$ and $Q$, so that now we indeed have $\mathcal{S}(\bar{\Sigma}) / N_{S}=G^{P}$.

How can such a different behaviour be explained? Formally, a major difference between handles and irreducible primes is that for handles we have the possibility to slide only their ends rather than the whole prime. This results is the difference of (5.3d) and (5.5c): a conjugation on generators, like in (5.5c), can be generated from (5.3) (sliding both ends) but a mere left or right multiplication on generators, like in (5.3d), cannot be generated from (5.5). This suggests that the presence of handles is responsible for the particle group not being a quotient. In the next section we report some work that proves this conjecture.

6. The General Case. We recall that in the general case $\pi_{1}(\bar{\Sigma})$ is a free product of the groups $\pi_{1}\left(P_{i}\right)$ and some $\mathbf{Z}$ 's. $\mathcal{S}(\bar{\Sigma})$ is to be built from $\operatorname{ker}\left(h_{\infty}(\bar{\Sigma})\right)$ and $\operatorname{Im}\left(h_{\infty}(\bar{\Sigma})\right) \subseteq$ $\operatorname{Aut}\left(\pi_{1}(\bar{\Sigma})\right)$. The reason why we can make statements about the general case lies in the happy fact that we are explicitly given a presentation for the automorphism group of free products. We use the form given in [18] (called the Fuks-Rabinovitch presentation), in which the generators of $\operatorname{Aut}\left(\pi_{1}(\bar{\Sigma})\right)$ are given by those of $\operatorname{Aut}\left(\pi_{1}\left(P_{i}\right)\right)$ and others which correspond to spin-, exchange-, and slide-diffeomorphisms. The lack of surjectivity of $h_{\infty}(\bar{\Sigma})$ on $\operatorname{Aut}\left(\pi_{1}(\bar{\Sigma})\right)$ resides entirely in the lack of surjectivity of its restrictions $h_{\infty}\left(\Pi_{i}\right)$ : $\mathcal{S}\left(\Pi_{i}\right) \rightarrow \operatorname{Aut}\left(\pi_{1}\left(\Pi_{i}\right)\right)$. For example, using Proposition 2, we may infer surjectivity if $\bar{\Sigma}$ is a connected sum of handles and sufficiently large $K(\pi, 1)$ primes. Since these are all HI-primes, we can combine this with the first example of the previous section and infer

THeOrem 3. Let $\bar{\Sigma}$ be a connected sum of $l$ handles and $n$ sufficiently large $K(\pi, 1)$ primes. Then $\mathcal{S}(\bar{\Sigma})$ is an extension of $\operatorname{Aut}^{+}\left(\pi_{1}(\bar{\Sigma})\right)$ by $\prod_{i=1}^{n+l} \mathbf{Z}_{2}$. 
Like in the $N$-handle example the extension would not be difficult to describe. The $\mathbf{Z}_{2}$ 's correspond to twists and rotations which commute with other internal diffeomorphisms and slides, but are acted upon by exchanges in the obvious way. Together with the explicit presentation of $\operatorname{Aut}\left(\pi_{1}(\bar{\Sigma})\right)$ this is then sufficient information to write down a presentation of $\mathcal{S}(\bar{\Sigma})$.

We now address the question posed at the end of the previous section. To do this one has to explicitly go through the Fuks-Rabinovitch relations as given in [18]. We will not repeat them here but rather refer the reader to the article by McCullough and Miller. We shall adopt their numbering of the relations. Amongst these relations are four of particular interest for us whose implications we now wish to explain:

Relation 30: Given any connected sum containing (amongst possible other primes) two handles $H_{i}, H_{j}$ and another prime $\Pi_{k}$ (which may be a handle or not), then slides of a handle-end of $H_{j}$ through $\Pi_{k}$ can be written as a commutator of slides.

Relation 47: Given any connected sum containing (amongst possible other primes) a prime $\Pi_{i}$ (which may be a handle or not), a handle $H_{j}$, and an irreducible prime $P_{k}$, then any slide of $P_{k}$ through $\Pi_{i}$ can be written as a commutator of slides.

These two observations imply

THEOREM 4. If $\bar{\Sigma}$ contains at least three handles then slides generate a perfect subgroup $N_{S}$ of $\mathcal{S}(\bar{\Sigma})$.

This generalizes our observation concerning slides made on the first example above.

Relation 31: Given any connected sum containing (amongst possible other primes) two handles $H_{i}$ and $H_{j}$, then a spin of $H_{j}$ followed by an exchange of $H_{i}$ and $H_{j}$ is generated by slides of handle-ends of $H_{i}$ through $H_{j}$ and of $H_{j}$ through $H_{i}$.

Relation 48: Given any connected sum containing (amongst possible other primes) an irreducible prime $P_{i}$ and a handle $H_{j}$, then internal slides of $P_{i}$ are generated by external slides of $P_{i}$ through $H_{j}$ and ends of $H_{j}$ through $P_{i}$.

General Observation: The last two relations are the only ones that imply additional relations for non-slide generators when the slides generators are set equal to the identity E. This implies:

THEOREM 5. If $\bar{\Sigma}$ contains no handle in its prime decomposition, then $\mathcal{S}(\bar{\Sigma})) / N_{S} \cong$ $G^{P}$. In fact, $\mathcal{S}(\bar{\Sigma})$ is a semi-direct product of $N_{S}$ with $G^{P}$.

Note that this implies in particular that all (irreducible) representations of $G^{P}$ extend to (irreducible) representations of $\mathcal{S}(\bar{\Sigma})$. We can now understand our two examples in the previous section as extreme cases for the last two theorems.

\section{References}

[1] A. Ashtekar, J. Lewandowski, D. Marolf, J. Mourão, and T. Thiemann, Quantization of diffeomorphism invariant theories with local degrees of freedom, J. Math. Phys. 36 (1995), 6456-6493; gr-qc/9504018.

[2] H. S. M. Coxeter and W. O. J. Moser, Generators and relations for discrete groups (second edition), Springer Verlag, Berlin-Göttingen-Heidelberg-New York, 1965. 
[3] A. E. Fischer, Resolving the singularities in the space of Riemannian geometries, J. Math. Phys. 27 (1986), 718-738.

[4] J. Friedman and D. Witt, Homotopy is not isotopy for homeomorphisms of 3-manifolds, Topology 25 (1986), 35-44.

[5] D. Giulini, Asymptotic symmetry groups of long-ranged gauge configurations, Modern Phys. Lett. A 10 (1995), 2059-2070.

[6] D. Giulini, On the configuration space topology in general relativity, Helv. Phys. Acta 68 (1995), 87-111.

[7] D. Giulini, Quantum mechanics on spaces with finite fundamental group, Helv. Phys. Acta 68 (1995), 438-469.

[8] D. Giulini, What is the geometry of superspace?, Phys. Rev. D (3) 51 (1995), 5630-5635.

[9] D. Giulini, 3-Manifolds for relativists, Internat. J. Theoret. Phys. 33 (1994), 913-930.

[10] D. Giulini and J. Louko, Diffeomorphism invariant states in Wittens $2+1$ quantum gravity on $R \times T^{2}$, Classical Quantum Gravity 12 (1995), 2735-2745.

[11] D. Giulini and J. Louko, Theta-sectors in spatially flat quantum cosmology, Phys. Rev. D (3) 46 (1992), 4355-4364.

[12] A. Hatcher, Homeomorphisms of sufficiently large $P^{2}$-irreducible 3-manifolds, Topology 15 (1976), 343-347.

[13] J. Hempel, 3-Manifolds, Ann. of Math. Stud. 86, Princeton University Press, 1976.

[14] H. Hendriks, Application de la théorie d'obstruction en dimension 3, Bull. Soc. Math. France Mém. 53 (1977), 81-195.

[15] H. Hendriks and D. McCullough, On the diffeomorphism group of a reducible 3manifold, Topology Appl. 26 (1987), 25-31.

[16] D. McCullough, Mappings of reducible manifolds, in: Geometric and Algebraic Topology, Banach Center Publ. 18 (1986), 61-76.

[17] D. McCullough, Topological and algebraic automorphisms of 3-manifolds, in: Groups of Homotopy, Equivalences and Related Topics, R. Piccinini (ed.), Lecture Notes in Math. 1425, Springer, Berlin, 1990, 102-113.

[18] D. McCullough and A. Miller, Homeomorphisms of 3-manifolds with compressible boundary, Mem. Amer. Math. Soc. 344 (1986).

[19] J. Milnor, Introduction to algebraic K-theory, Ann. of Math. Stud. 72, Princeton University Press, 1971.

[20] S. P. Plotnick, Equivariant intersection forms, knots in $S^{4}$, and rotations in 2-spheres, Trans. Amer. Math. Soc. 296 (1986), 543-575.

[21] D. Witt, Symmetry groups of state vectors in canonical quantum gravity, J. Math. Phys. 27 (1986), 573-592.

[22] D. Witt, Vacuum space-times that admit no maximal slice, Phys. Rev. Lett. 57 (1986), 1386-1389. 